\title{
Validation of a High-Temperature Experimental Setup for Thermoelectric Characterization of Novel Materials
}

\author{
Eduardo E. Castillo, PhD., Jonathan Pierce \\ Universidad Ana G. Méndez, Puerto Rico, ecastillo@uagm.edu, jpierce1@email.uagm.edu
}

\begin{abstract}
This research validates an experimental setup to measure the thermal and electrical resistances of novel thermoelectric materials. The efficiency of these materials increases with temperature, with maximum values around $600{ }^{\circ} \mathrm{C}$. New experimental setups that take account of the heat transfer losses and material properties at that high temperature are needed. The sample for this validation is a thermoelectric generator, TG12$8 L$ from Marlow Industry. A low AC current ( $5 \mathrm{~mA}$ ) was applied to measure the electrical resistance, minimizing the Joule heating and Peltier effects. The thermal resistance was calculated by using a 1-D steady state model. For that model: 1) the setup was enclosed in a vacuum chamber to minimize the convection heat losses, while a ceramic heater was used as the heat source; 2) to improve the thermal contact with the sample, two thin aluminum plates sandwiched the sample and were used as a heat spreader; 3) A copper block with K-type thermocouples for heat flux measurements were used. Electrical resistance around 1.5 ohms for the sample was calculate from the voltage versus current graph, with excellent agreement with the manufacturer values. As expected, the electrical resistance was increasing with temperature. The thermal resistance was calculated as $1.503{ }^{\circ} \mathrm{C} / W$, which is higher than the manufacturer values. This difference could be due to radiation heat transfer and other energy losses, and also due to the unsteady measurements in the big thermal mass in the setup. Future setups will minimize the heat transfer parasitic effects and time for steady state.
\end{abstract}

Keywords-- Thermoelectric measurements, Thermoelectric generator, Seebeck effect, Figure of merit.

\section{INTRODUCTION}

Thermoelectric effect is the direct conversion of temperature gradient into electric voltage and the opposite process of using electricity to drive the heat flow and generate temperature differences. This effect can be employed to generate electricity from heat, measure temperature, and pump the heat from/to an object. Thermoelectric power generators are employed in applications with available heat sources such as cars, electronic wrist watches, temperature warning systems, active transponders and waste heat harvesting systems [1]-[3]. Thermoelectric heat pumps for thermal management applications are essential in modern optical telecommunications satellite and spacecraft industry, bioanalytical instrumentation, high performance liquid chromatography and refrigerators/freezers [4]-[6].

The thermoelectric energy conversion efficiency is determined by the dimensionless figure of merit $\mathrm{ZT}=\alpha^{2} \mathrm{~T} / \rho k$, where $\alpha$ is the Seebeck coefficient, $\mathrm{T}$ is the absolute temperature, $\rho$ is the electrical resistivity and $k$ is the thermal conductivity. While bulk thermoelectric materials have ZT $\sim$, nanostructured thermoelectric materials have received much

Digital Object Identifier (DOI):

http://dx.doi.org/10.18687/LACCEI2020.1.1.325

ISBN: 978-958-52071-4-1 ISSN: 2414-6390 attention during the last decade due to their potential for enhanced thermoelectric energy conversion efficiency. Most of nanostructured materials of high ZT values are films on substrate. Also, the figure of merit of these materials increase with the temperature, reaching a maximum close to $600{ }^{\circ} \mathrm{C}$. Fast and reliable thermoelectric properties characterization of nanostructured materials at high temperature is of high interest [3], [5]. The energy conversion of these materials is greatly influenced by three main effects, the Seebeck effect, Peltier effect and joule heating.

The Seebeck effect is the direct conversion of temperature gradient into electricity, which is named after German physicist Thomas Johann Seebeck. As shown in Fig. 1, if two materials $a$ and $b$ (typically metal and semiconductor) form junctions, the temperature difference across the two junctions generates a direct electric voltage, which can light a bulb.

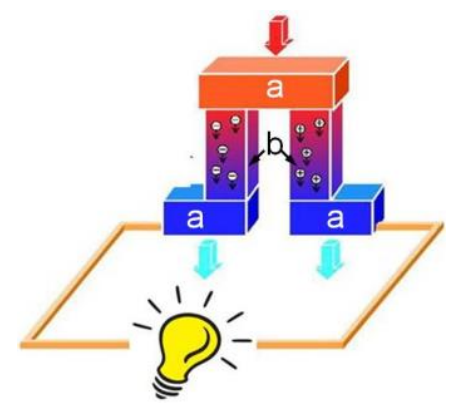

Fig. 1 A thermoelectric power generator utilizing Seebeck effect in metal (material a) and semiconductor (material b) junctions.

The Seebeck coefficient, $\alpha$, key parameter in thermoelectric materials, is defined as the amount of voltage generated per unit temperature difference,

$$
\alpha=-\frac{\Delta V}{\Delta T}
$$

where $\Delta \mathrm{V}$ is the generated Seebeck voltage and $\Delta \mathrm{T}$ is the temperature difference across the material. In a closed circuit, the Seebeck voltage induced by temperature differences can drive electronic devices and work as thermoelectric power generator.

The Peltier effect is the presence of heat flow and thus temperature differences driven by electrical current at a junction of two different materials and is named after French physicist Jean-Charles Peltier. Fig. 2 shows the Peltier effect, which can be used for solid-state refrigeration. The Peltier effect is based upon the differences in thermal energy per 
charge carrier carried in different materials, which is defined as the Peltier coefficient.

The Peltier effect can be used for solid-state cooling, also called thermoelectric cooling, which has no moving part or gas emission.

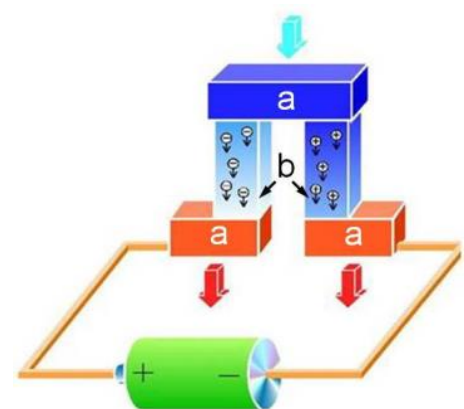

Fig. 2 A thermoelectric refrigerator utilizing Peltier effect in metal (material a) and semiconductor (material b) junctions.

Finally, Joule heating is the effect due to the opposition to the pass of the electrical current. The Joule heating is equal to the electrical resistance of the material times the electrical current to the square. This heating is one of most important conditions that limited the advance of technologies like the heating of the microprocessors in the computer and of course, also the applications of the thermoelectrics.

\section{EXPERIMENTAL SETUP}

Fig. 3 shows a photo of the thermoelectric module used for the experiment. This module, TG12-8 Marlow Industry, Inc. [7], has an AC resistance between 1.36-1.69 ohms, based on a cold side at $27 \pm 2{ }^{\circ} \mathrm{C}$.

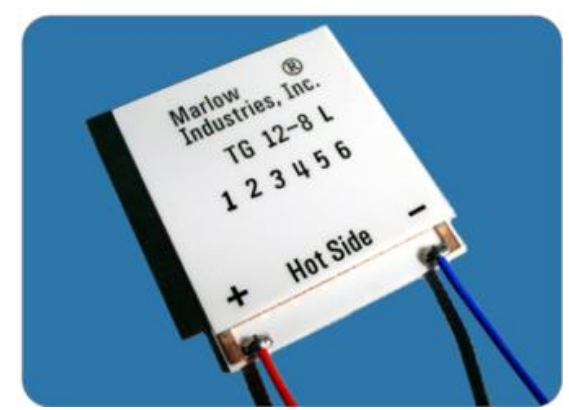

Fig. 3 Thermoelectric module used for the validation [7].

Other parameters of this module are based on a cold side at $50{ }^{\circ} \mathrm{C}$ and hot side at 110,170 , or $230{ }^{\circ} \mathrm{C}$; as is shown in Table 1. Another important property of this module is the thermal resistance, which oscillate between 1.13 to $1.20^{\circ} \mathrm{C} / \mathrm{W}$. Table 1 shows a detailed list of properties for the TG12-8 Marlow Industry, Inc.

\section{A. Electrical Setup}

Two different electrical configurations where prepared for the electrical measurements. In the first configuration, in order to measure the electrical resistance of the module, an $\mathrm{AC}$ current was used to avoid the Seebeck voltage generated by a DC current. Also, low current (in the order of $\mathrm{mA}$ ) was used to minimize the Joule heating. For the second configuration, as the temperature of the system increase, the generated DC Seebeck voltage caused by the temperature difference in the module was measured.

Table 1 Thermoelectric Properties of the TG12-8 module.

\begin{tabular}{|c|c|c|c|}
\hline \multicolumn{2}{|c|}{ Typical Performance Value } & & \\
\hline Cold Side Temperature, $\mathrm{T}_{\mathrm{c}}\left({ }^{\circ} \mathrm{C}\right)$ & $27 \pm 2$ & & \\
\hline AC Resistance (Ohms) & $1.36-1.69$ & & \\
\hline Device $\mathrm{ZT}_{\mathrm{c}}$ & 0.73 & & \\
\hline \multicolumn{4}{|c|}{ Thermoelectric Properties } \\
\hline Hot Side Temperature $\left({ }^{\circ} \mathrm{C}\right)$ & 230 & 170 & 110 \\
\hline Cold Side Temperature $\left({ }^{\circ} \mathrm{C}\right)$ & 50 & 50 & 50 \\
\hline Optimum Efficiency, $\eta(\%)$ & 4.97 & 4.08 & 2.39 \\
\hline Optimum Power (W) & 7.95 & 4.17 & 1.19 \\
\hline Optimum Voltage & 5.25 & 3.65 & 1.86 \\
\hline $\begin{array}{l}\text { Load Resistance for Opt } \eta \\
(\Omega)\end{array}$ & 3.46 & 3.2 & 2.9 \\
\hline Open Circuit Voltage, VOC (V) & 9.43 & 6.48 & 3.27 \\
\hline Closed Circuit Current (A) & 3.38 & 2.6 & 1.48 \\
\hline Thermal Resistance $\left({ }^{\circ} \mathrm{C} / \mathrm{W}\right)$ & 1.13 & 1.17 & 1.2 \\
\hline
\end{tabular}

For the first configuration, a function generator is used to send an AC current across the thermoelectric module (TE), and a series resistance is used to determine the electrical current, as shown in Fig. 4. Then, the AC voltage drop across the TE and across the series resistance were measured. These measurements of the TE electrical resistance were performed very fast, in order to avoid changes due to the temperature of the system.

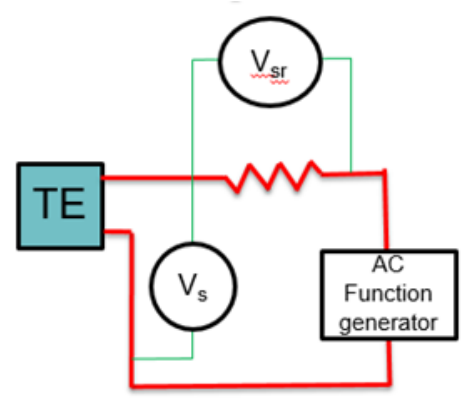

Fig. 4 Electrical configuration to the Thermoelectric.

For the second configuration, as the temperature of the system increase, the DC Seebeck voltage generated in the TE was measured; at the same time as the thermal measurements. Also, the series resistance was used as an electrical load, and the power generated could be determinate. 
Fig. 5 shows the instruments used for the electrical and thermal setups: TR, a Stanford Research System thermocouple reader (Model SR630); FG, an Agilent function generator (Model 33220A); and DMM, an Agilent digital multimeter (Model 34970A). The thermocouple reader was used to measure the temperatures of the hot and cold side of the TE, and the top and bottom temperatures of the heat flux sensor, $T_{h}, T_{c}, T_{t}$, and $T_{b}$, respectively. The function generator was used to supply a low AC current to avoid the Joules heating and the Peltier's effects. The digital multimeter was used to measure the: DC Seebeck voltage, DC Voltage Sample, and series resistor.

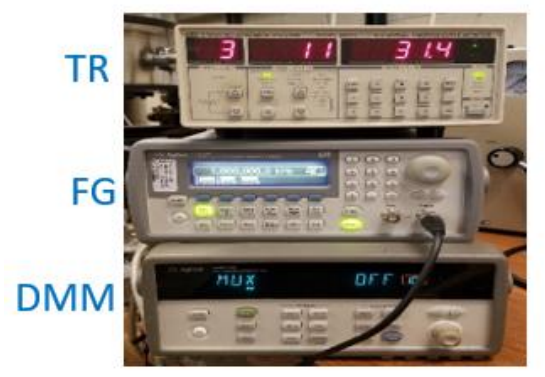

Fig. 5 Instruments used for the electrical and thermal setups: TR, Stanford Research System thermocouple reader (Model SR630); FG, Agilent function generator (Model 33220A); and DMM, Agilent digital multimeter (Model 34970A)

\section{B. Thermal Setup}

Fig. 6 shows a colored diagram of the main components used for the thermal setup. These components were: a heater, two thin aluminum plates, the thermoelectric module, a heat flux sensor, a heat sink, thermocouples, and a vacuum chamber. The power of the ceramic heater was controlled by a rheostat and capable of reaching up to $1000^{\circ} \mathrm{C}$. This type of heater was selected for this study because it is easily portable and gives off a great deal of heat from a small design. Also, these heaters are more energy efficient and safer than many other heaters.

The two aluminum plates were used to spread the heat uniformly to the TE and to improve the thermal contact with it. The thermoelectric module is the TG12-8, Marlow Industries Inc, as mentioned before. The output of the thermoelectric was connected as shown before in the section of electrical setup. The copper heat flux sensor was used to measure the 1-D heat flux that passes to the heat sink, having the dimensions of $12.7 \mathrm{~mm} \times 27.1 \mathrm{~mm} \times 60 \mathrm{~mm}$ for thickness, width and length, respectively.

Thermocouples type $\mathrm{K}$ were used to measure the temperatures: $T_{h}, T_{c}, T_{t}$, and $T_{b} ; T_{h}$ and $T_{c}$ of the hot and cold surfaces of the thermoelectric, respectively. On the other hand, $T_{t}$ and $T_{b}$ are the top and the bottom temperatures of the heat flux sensor, with $50.8 \mathrm{~mm}$ of separation. Finally, the system was set inside a vacuum chamber with a pressure of $1 \times 10^{-4}$
Torr, which was employed to minimize the convection heat losses to the surrounding area. All these instruments mentioned before were connected to a computer and all the data was gathered using the LabView program. Fig. 7 shows a photo of the actual setup being used.

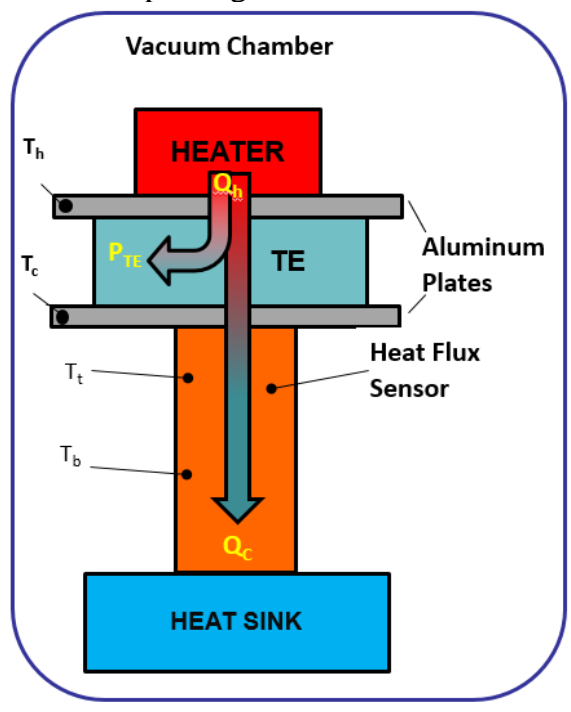

Fig. 6 Schematic of the thermal system.

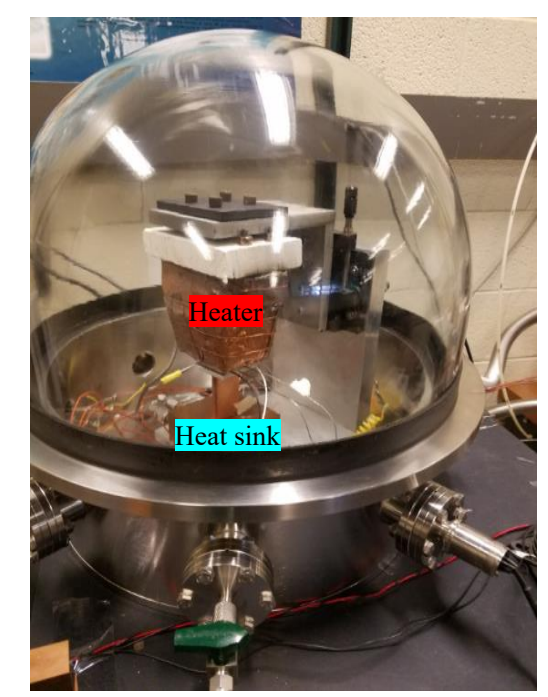

Fig. 7 Actual system inside the vacuum chamber.

\section{RESULTS}

As mentioned before, the system was initially set to calculate the electrical resistance of the TE without any heating or at room temperature $\left(30^{\circ} \mathrm{C}\right)$. Then, to calculate the dependence of the electrical and thermal resistances with temperature, the heater was set at several levels of power (from 40 to 70 percent) until reach a steady state. The following sections illustrate the results of the electrical and thermal parts. 


\section{A. Electrical Setup}

The initial measurement was made to determine the electrical resistance without heating. The function generator was configured to produce a sine wave with $1000 \mathrm{~Hz}$ of frequency and an amplitude from 0.4 to $1.2 \mathrm{~V}$ in four steps, which produce an $\mathrm{AC}$ current in the sample approximately from 2.5 to $9.0 \mathrm{~mA}$. Fig. 8, shows the Voltage vs Current graph for the room temperature measurements. As it shows, the graph is perfectly linear and the electrical resistance of the $\mathrm{TE}$ was calculated as $\mathrm{Re}=1.5983 \Omega$; which lines up with the manufacturer specifications.

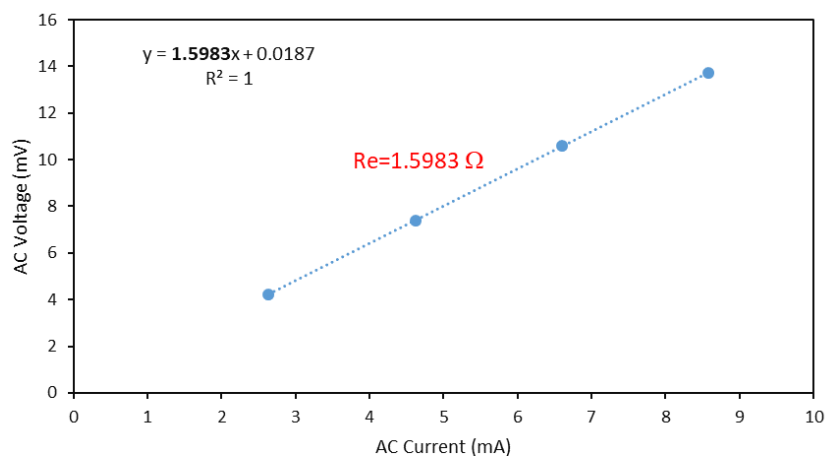

Fig. 8 Voltage vs Current for the AC measurements of the electrical resistance at room temperature.

Then, when the heater was on, these $\mathrm{AC}$ voltages were applied again to measure the electrical resistance as function of the average temperature between $T_{c}$ and $T_{h}$. Fig. 9 shows the $T_{c}$ and $T_{h}$ temperatures as a function of the measurement number, where each measurement takes about one minute. Initially, the temperatures decrease a little due to the vacuum chamber being closed. Then, after measurement 50 approximately, the heater was turned on and the graphs show five additional segments with different slopes, each one represents the changes in the power supplied by the heater. Also, as we can see, the temperature of the cold side of the thermoelectric, $T_{c}$, does not remain constant, meaning that the heat sink does not have enough cooling capacity.

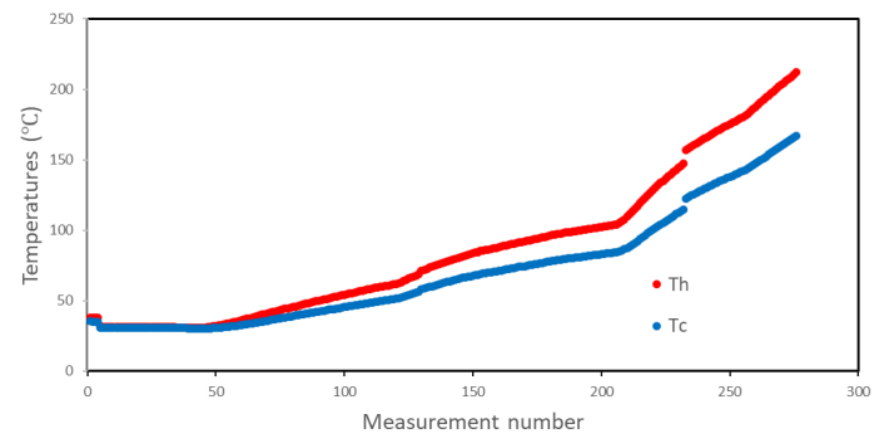

Fig. 9 Temperature of the hot and cold side of the TE as a function of the measurement number. The different slopes correspond to the changes in the heater power.
Future experiments will need to circulate cold water from the outside to the internal circuit in the copper block, that works as a heat sink, to keep a constant temperature.

Finally, Fig. 10, shows the electrical resistance of the TE as a function of the average temperature of the two sides. As it is seen, the two initial parts show a linear relationship, where the temperature coefficient of resistance (TCR) can be calculated from the slope. More data needed to be analyzed to interpret the jumps in this graph as well as the rapid increase in the logarithmic part.

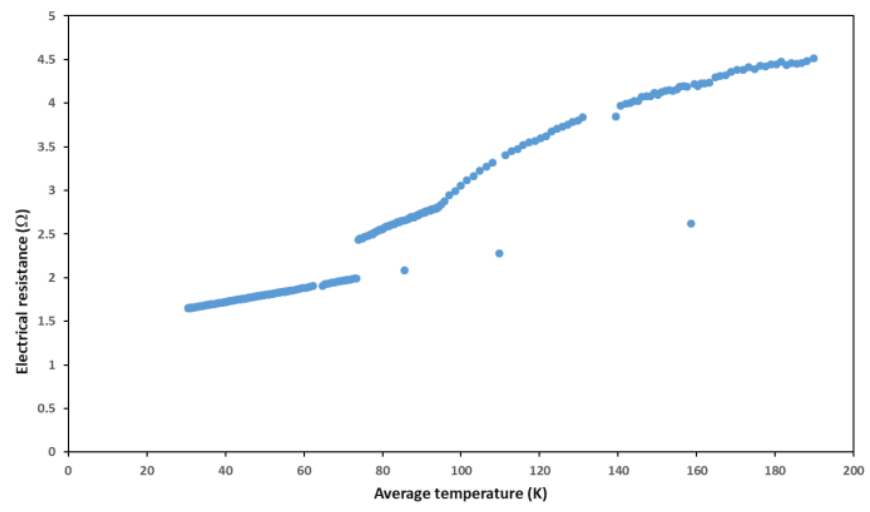

Fig. 10 Electrical resistance of the TE as a function of the average temperature of the sides.

\section{B. Thermal Setup}

To calculate the dependence of the electrical and thermal resistances with temperature, the heater was set at several levels of power (from 40 to 70 percent) until it reached a steady state. Due to the big mass of the whole system, for each power in the heater used, it took a couple hours to reach the steady state. Then, for each steady state, the electrical resistance of the TE was measured as mentioned before and immediately the temperatures were measured for the thermal analysis.

Fig. 11 shows the temperature difference and the generated Seebeck voltage as a function of the measurement number. The initial descending part of the graph is after the vacuum chamber was closed and without heating, when the two temperatures of the systems start to be similar. Also, as we can see, the generated Seebeck voltage followed the behavior of the temperature difference. 


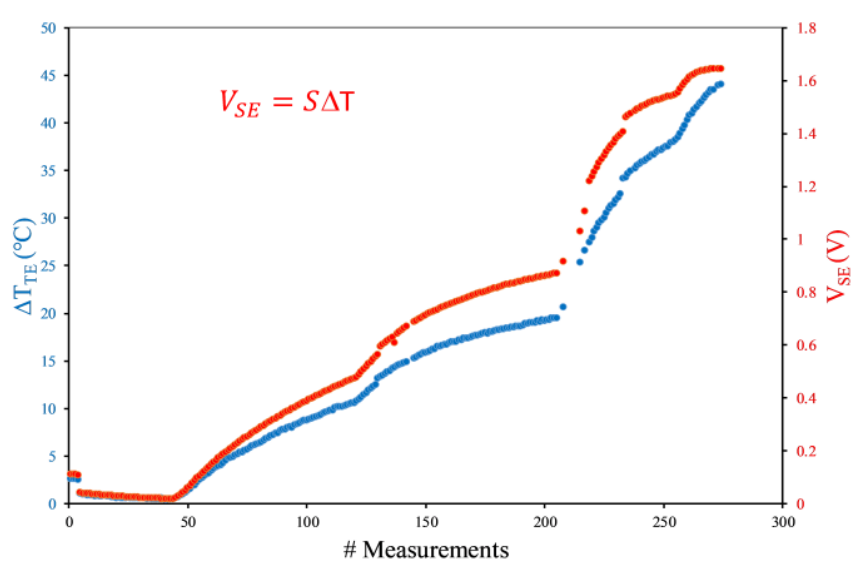

Fig. 11 Temperature difference and Seebeck voltage as a function of the measurement number.

Fig. 12 shows the Seebeck voltage as a function of the temperature difference. Although the graph shows some outliers points, maybe due to contact problems, the graph shows a very linear tendency. Then, after subtracting those points, the Seebeck coefficient of the TED could be calculated as $43.8 \mathrm{mV} / \mathrm{K}$, unfortunately, the manufacturer does not provide this value to compare.

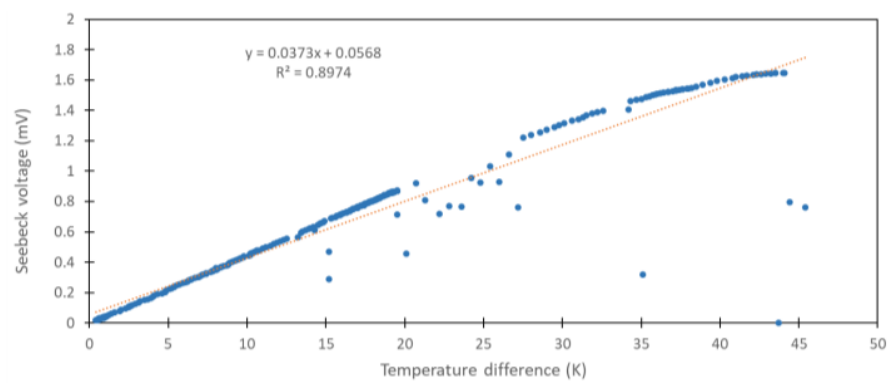

Fig. 12 Seebeck voltage as a function of the temperature difference. the graph shows some outliers points maybe due to contact problems. The slope of the graph represents the Seebeck coefficient of the TE.

Finally, the heat transfer rate to the heat sink could be calculated from the classical 1-D heat conduction in the heat flux sensor as:

$$
Q c=\frac{k_{s} A}{t}\left(T_{t}-T_{b}\right)
$$

where $Q c$ represents the heat transfer rate through the cold side of the TE, $k$ is the thermal conductivity of the copper block, used as $400 \mathrm{~W} / \mathrm{m} . \mathrm{K}, A$ is the cross sectional area of the copper block and $t$ is the distance between the two temperatures $T_{t}$ and $T_{b}$. The equation (2) assumes that there is no radiation and convection from the heater or from the rest of the setup. A radiation shield will be necessary for higher temperatures to be used in this work.

The behavior of the heat flux is very similar to the temperatures shown in Fig. 9; but with values from zero to $30.6 \mathrm{~W}$. Fig. 13 shows the temperature difference in the TE as a function of the heat transfer rate. The graph shows a relationship and from the slope the thermal resistance of the device can be calculated as $\mathrm{R}_{\mathrm{th}}=1.503{ }^{\circ} \mathrm{C} / \mathrm{W}$ which is higher compared with the $1.13-1.2{ }^{\circ} \mathrm{C} / \mathrm{W}$ from the manufacturer; but our value is based on the heat transfer in the cold side of the thermoelectric. In addition, after decreasing the energy loses from the setup, the thermal resistance of the TE will be better.

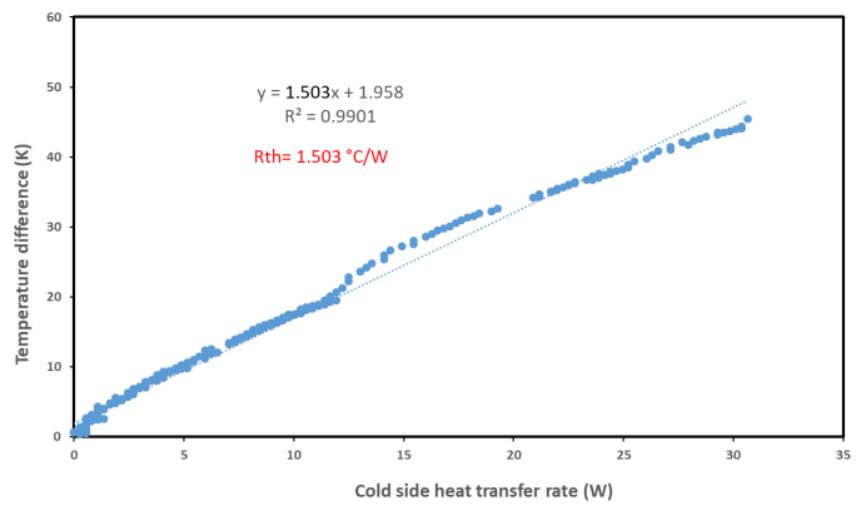

Fig. 13 Temperature difference in the TE as a function of the heat transfer rate in the cold side of the TE.

\section{CONCLUSIONS}

Using the thermoelectric, TG12-8 from Marlow Industry, Inc., as a calibration sample, this work was able to measure the electrical and thermal resistances of the module as a function of the temperature. Due to the temperature restrictions of the module, the system was only raised until $250{ }^{\circ} \mathrm{C}$, but the main objective is going until $600{ }^{\circ} \mathrm{C}$. The results for the electrical resistance were from 1.5 until $5 \Omega$ which is in good agreement with the manufacturer data.

The results for the thermal resistance was $1.503{ }^{\circ} \mathrm{C} / \mathrm{W}$ which is higher that the manufacturer, but still pretty good for thermal measurements at high temperature.

In addition, with this setup the Seebeck coefficient of the TE device was measured as $43.8 \mathrm{mV} / \mathrm{K}$ and future works will be exploring to calculate the temperature coefficient of resistance of the device.

\section{FUTURE WORK}

As was mention before, the measurements were only until $250{ }^{\circ} \mathrm{C}$. Then a new TE device with higher temperature applications will be used. In addition, several improvements will be performed in this setup. First, a radiation shield will be used in the heater to minimize the radiation to the sample and to the heat flux sensor. Longer time before taking the measurements will be necessary to ensure the steady state in the system. Finally, cold water will be used in the heat sink to keep a constant temperature. 


\section{ACKNOWLEDGMENT}

The authors thanks to Dr Theodorian Borca-Tascuic at Rensselaer Polytechnic Institute (RPI), Troy NY, for his unconditional support and mentoring. Also, to the laboratory staff for their hands-on assistance. On the other hand, the authors acknowledge to the Universidad Ana G. Méndez for their support in this research.

\section{REFERENCES}

[1] F. Xiao, C. Hangarter, B. Yoo, Y. Rheem, K. H. Lee, and N. V. Myung, "Recent progress in electrodeposition of thermoelectric thin films and nanostructures," Electrochim. Acta, vol. 53, no. 28, pp. 8103-8117, 2008, doi: 10.1016/j.electacta.2008.06.015.

[2] Y. Le Maho, "Contents of Volume," Autom. Constr., vol. 20, no. 8, pp. 1260-1264, 2011, doi: 10.1016/S0926-5805(11)00187-7.

[3] K. A. Cook-Chennault, N. Thambi, and A. M. Sastry, "Powering MEMS portable devices - A review of non-regenerative and regenerative power supply systems with special emphasis on piezoelectric energy harvesting systems," Smart Mater. Struct., vol. 17, no. 4, 2008, doi: 10.1088/0964-1726/17/4/043001.

[4] C. LaBounty, A. Shakouri, and J. E. Bowers, "Design and characterization of thin film microcoolers," J. Appl. Phys., vol. 89, no. 7, pp. 4059-4064, 2001, doi: 10.1063/1.1353810.

[5] M. S. Dresselhaus et al., "New directions for low-dimensional thermoelectric materials," Adv. Mater., vol. 19, no. 8, pp. 10431053, 2007, doi: 10.1002/adma.200600527.

[6] E. E. Castillo, C. L. Hapenciuc, and T. Borca-Tasciuc,

"Thermoelectric characterization by transient Harman method under nonideal contact and boundary conditions," Rev. Sci. Instrum., vol. 81, no. 4, 2010, doi: 10.1063/1.3374120.

[7] "No Title." https://www.marlow.com/products/power-generators. 